\title{
Breastfeeding in Coronavirus Disease 2019 (COVID-19): Position Statement of Indian Academy of Pediatrics and Infant and Young Child Feeding Chapter
}

\author{
Ketan Bharadva, ${ }^{1}$ Roopa M Bellad, ${ }^{2}$ Satish Tiwari, ${ }^{3}$ R Somasekar, ${ }^{4}$ Mrudula Phadke, ${ }^{5}$ Uday Bodhankar, ${ }^{6}$ \\ Akash Bang, ${ }^{7}$ Aarti Avinash Kinikar, ${ }^{8}$ HB Mallikarjuna,,${ }^{9}$ Jayant Shah, ${ }^{10}$ Omesh Khurana, ${ }^{11}$ \\ D Gunasingh, ${ }^{12}$ GV Basavaraja, ${ }^{13}$ Remesh Kumar, ${ }^{14}$ Piyush Gupta ${ }^{15}$ \\ From ${ }^{1}$ Department of Pediatrics, Masoom Children's Hospital, Surat, Gujarat; ${ }^{2}$ KAHER J N Medical College, Belagavi, \\ Karnataka; ${ }^{3}$ Dr PDM Medical College, Amravati, Maharashtra; ${ }^{4}$ Sree Balaji Medical College and Hospital, Chrompet, Chennai, \\ ${ }^{5}$ Former VC MUHS and Advisor UNICEF and NHM; ${ }^{6}$ Deputy Chairperson Commonwealth Professional Health Alliance - UK; \\ ${ }^{7}$ AIIMS, Nagpur, Maharashtra; ${ }^{8}$ BJ Government Medical College and Sassoon General Hospital, Pune, Maharashtra; 9 \\ MS Ramaiah Memorial Hospital, Bangalore, Karnataka; ${ }^{10}$ Shaishav Hospital, Nandurbar, Maharashtra; ${ }^{11}$ CCM Medical College, \\ Durg, Chhattisgarh; ${ }^{12}$ Arumai Medical College, Tiruvannamalai; ${ }^{13}$ IGICH, Bengaluru, Karnataka; ${ }^{14}$ President Elect IAP, \\ Apollo Adlux Hospital, Cochin, Kerala, ${ }^{15}$ President, Indian Academy of Pediatrics, Mumbai. \\ Correspondence to: Dr Roopa M Bellad, Professor Pediatrics and Director Academic Affairs, KAHER, JN Medical College, \\ Belagavi, Karnataka. belladroopa5@gmail.com
}

Justification: Recent research has provided evidence for lack of transmission of SARS-CoV-2 through human milk and breastfeeding. Updating the practice guidelines will help in providing appropriate advice and support regarding breastfeeding during the coronavirus 2019 (COVID-19) pandemic. Objectives: To provide evidence-based guidelines to help the healthcare professionals to advise optimal breastfeeding practices during the COVID-19 pandemic. Process: Formulation of key questions was done under the chairmanship of President of the IAP. It was followed by review of literature and the recommendations of other international and national professional bodies. Through Infant and Young child (IYCF) focused WhatsApp group opinion of all members was taken. The final document was prepared after the consensus and approval by all members of the committee. Recommendations: The IYCF Chapter of IAP strongly recommends unabated promotion, protection and support to breastfeeding during the COVID-19 pandemic with due precautions.

Keywords: Guidelines, Human milk, Lactation, SARS-CoV-2, Support.

Published online: November 22, 2021; PII: S097475591600373

T he risk of transmission of the severe acute respiratory syndrome coronavirus 2 (SARSCoV-2) from coronavirus disease 2019 (COVID-19) positive mother to the infant through breastfeeding; and safety of the infants in case of discontinuation of breastfeeding are the major concerns of the healthcare providers and community during the pandemic.

In May, 2020, World Health Organization(WHO), United Nations Children's Fund (UNICEF), Government of India (GOI), Indian Council of Medical Research (ICMR), Centers for Disease Control and Prevention USA (CDC), National Neonatology Forum of India (NNF), Federation of Obstetric and Gynaecological Societies of India(FOGSI), Breastfeeding Promotion Network of India (BPNI) and Infant and Young Child Feeding Chapter of Indian Academy of Pediatrics (IYCFIAP), recommended early initiation of exclusive breastfeeding for first 6 months and breastfeeding with proper complementary feeding until 2 years and beyond, while using necessary precautions for infection prevention and control, in infants born to mothers with suspected or confirmed COVID-19 [2-9]. These recommendations were based on the health benefits associated with breastfeeding for both mother and child; and relatively mild or asymptomatic illness experienced by infants reported so far. But the rising concerns and insufficient available evidence of mother to infant transmission of SARS-CoV-2 through breastfeeding, contributed to the differing guidelines and recommendations from public health agencies, and international and national authorities [10,11].

Infant feeding practices like avoiding of breastfeeding, promoting artificial feeding and separation of mother and infant has implications on the growth and development and long term adverse health outcomes in the infants. Interruption of breastfeeding causes decrease in milk supply and mother needs help and counselling to restore it later. Hence stopping of breastfeeding should not be advised. India has a high newborn mortality rate and a third of all preterm births in the world [12], and ensuring adequate availability of human milk becomes an important 
intervention by the healthcare system for reducing neonatal and infant mortality and morbidity during the pandemic.

Since the last recommendations by the international and national agencies, recent research has provided newer evidence regarding transmission of SARS-CoV-2 from mother to child and through other infant feeding practices. Recent studies have reported increasing titers of antibodies to SARS-CoV-2 in the human milk following vaccination of the lactating mothers, thereby demonstrating the protection against COVID-19 to the mothers and their infants by vaccination $[13,14]$. Recently GOI has published a circular recommending administration of COVID-19 vaccines in breastfeeding women [15].

With the new available evidence and recent recommendation of vaccination of breastfeeding women by GOI, there is a need to review and update the guidelines to promote, protect and support breastfeeding during the pandemic. This will also help ameliorate the concerns among the frontline healthcare providers, pediatricians, obstetricians and nurses on whether they should encourage the infected mother to breast feed her baby.

\section{OBJECTIVES}

To provide evidence-based guidelines to help the healthcare professionals to advise optimal breastfeeding practices during the COVID-19 pandemic.

\section{PROCESS}

Formulation of key question was done under the chairmanship of the president of Indian Academy of Pediatrics. A committee of experts was formed by the chairman. It was followed by review of literature regarding risk of transmission and safety of promoting breastfeeding among mothers who are suspected or confirmed positive. The recommendations of other international and national professional bodies were also reviewed in detail. The available data was discussed in an Infant and Young Child Feeding-focused WhatsApp group. Opinion of all members was taken and the final document was prepared after consensus, which was approved by all members of the committee.

\section{RECOMMENDATIONS}

The recommendations are tabulated in $\mathbf{B o x} \mathbf{I}$.

\section{Breastfeeding and COVID-19}

Breastfeeding not only provides nutrition to the babies but is also benefecial to the mother and the baby in a variety of ways [16-18]. During the SARS-CoV-1 outbreak in 2003, lactoferrin in human milk was known to interact with heparin
Box I Indian Academy of Pediatrics, Infant and Young Child Feeding Chapter Recommendations

- Benefits of breastfeeding and $\mathrm{KMC}$ in providing nutrition and prevention of infections cannot be overlooked, which is of crucial importance in developing countries like India.

- Breastfeeding has specific benefits to the infants during COVID-19 pandemic and so encourage, promote, protect and support breastfeeding in all the babies during the pandemic.

- Mothers with suspect or confirmed COVID-19 should initiate early (within one hour after birth) and exclusive breastfeeding and KMC to their babies with due precautions to prevent and control COVID-19 during breastfeeding i.e. ,wearing of appropriate mask, proper washing /sanitizing of the hands before and after feeding session and routinely disinfecting and cleaning the high contact surfaces.

- Mother and baby should stay together as much as possible, unless she is too sick to take care, to have skin to skin contact, to feed their baby responsively and to have access to ongoing support when this is needed, while implementing infection control measures as above.

- If mother is ill, expressed human milk is to be fed to the baby by a care taker who is COVID-19 negative. All care takers should be taught to support mothers specifically in the breast milk expression and feeding the infant with EBM.

- All mothers and healthcare workers attending her should be taught skills of manual expression of breast milk by trained staff.

- For unfortunate cases of mother child separation due to severe illness of mother, mother must be helped to establish breastfeeding through specific re-lactation support.

- If mother's milk is not available, wet nursing (with proper safety procedural precautions) may be adopted or pasteurized donor human milk from a standard human milk bank or comprehensive lactation management center can be fed by the caregiver.

- We recommend the administration of COVID-19 vaccines to all women breastfeeding their infant.

- It is recommended that the lactating mothers and their families should be provided with psychosocial and technical support in confidence building and other aspects of breastfeeding especially in establishing/reestablishing and maintaining the milk supply. Also, counselling and extra support should be provided to the mothers and their family members, by dedicated trained counsellors if possible, regarding breastfeeding and education regarding COVID-19 prevention behaviors.

- Widespread dissemination of this guideline is recommended using various means and presentations, to healthcare workers and public in general. This is especially pertinent when healthcare facilities are disrupted in the pandemic, and IMS promotion lurks stealthily through many avenues. 
sulphate glycosaminoglycan (HSPG) cell receptors, interfering with the first anchoring sites of the virus on the cell, and thus preventing the initial contact between the SARS-CoV and host cells. Lactoferrin also blocks the interaction between spike viral protein and HSPC in an angiotensin-converting enzyme 2 (ACE2) receptor, which otherwise results in the full infection [19]. Presence of the oligosaccharides in human milk acts as a barrier to pathogens and as a prebiotic, promote synthesis of a healthy microbiota thereby preventing attachment of viruses to the mucosa and thus preventing infection [20]. Additional protective properties of human milk are provided by the transfer of maternal immune cells like macrophages, neutrophils and lymphocyte and secretory IgG and IgA to the breastfed infant. It also enhances the maturation of both innate and adaptive immune systems and thus protects the infants from infections [17]. Antibodies to SARS-CoV-2 with strong neutralizing capabilities have been isolated in human milk of COVID-19 positive mothers, suggesting active form of protection provided by human milk against COVID-19 [21,22]. Recently, IgA antibodies and other bioactive factors were detected in the human milk from COVID-19 positive mothers suggesting its key role in preventing life threatening infections during COVID-19 pandemic [23].

\section{Risk of Transmission}

There is limited but increasing evidence that human milk is not a source of SARS-CoV-2 infection to infants who are breast fed by COVID-19 positive mothers. Transmission of the virus though human milk is the major concern during the COVID-19 pandemic. Earlier studies have reported presence of the virus in human milk. However, these were small studies and case reports with low quality evidence, and failed to show the evidence that the virus was complete and or active in disease causation [21,24-30]. Recent larger prospective cohort studies have not detected SARS-CoV-2 in human milk samples collected from women with symptomatic infection $[22,31,32]$. Therefore, it cannot be concluded that mere presence of virus in the human milk can be directly related to the transmission of infection from mother to child and these studies confirm that SARS-CoV-2 can rarely get transmitted through human milk. This is further highlighted in recent systematic reviews and metaanalyses [16,17,29].

Recently, GOI and IAP-ACVIP have recommended to vaccinate all breastfeeding women against COVID-19 $[15,33]$. Studies have demonstrated that maternal vaccination results in high titers of antibodies to SARSCoV-2 and so is protective for the baby [13]. Based on this recent evidence of specific benefits of breastfeeding during the pandemic, with no chance of transmission of COVID-19 virus through breastfeeding, we need to continue to promote, protect and support breastfeeding.

\section{Separation of Mother and the Baby}

WHO guidelines (May 27, 2020) state not to separate mothers with suspected or confirmed COVID-19 unless the mother is too sick to care for her baby, and to provide skin to skin contact including kangaroo mother care. However, because of the insufficient available evidence, some national health agencies advised separation of infants from mothers with suspected or confirmed SARS-CoV-2 and avoidance of breastfeeding [10,11]. A recent study of a public health approach for deciding policy on infant feeding and mother-infant contact in the context of COVID-19 concluded that, deaths among infants affected by a policy of separation and not breastfeeding would be at least 67times greater than mortality potentially attributable to COVID-19 [34]. The survival benefits of breastfeeding substantially outweigh the very low reported CFR (case fatality rates) among infants with COVID-19. Separation of the mother and infant is therefore unnecessary and is potentially harmful [34]. Mothers should be enabled to stay together as much as possible with their babes, to have skinto-skin contact, to feed their baby responsively, and to have access to ongoing support when this is needed. Based on the clinical condition of mother and newborn, the decision may be taken on a case-to-case basis (Table I).

\section{Recommendations by Other Professional Bodies}

In May 2020, WHO updated its recommendation and advised early (within one hour of birth) and exclusive breastfeeding for first 6 months and then appropriate complementary feeding with breastfeeding until 2 years and beyond, while using necessary precautions for infection prevention and control in infants born to mothers with suspected or confirmed COVID-19. Mothers who were too ill to breast feed are advised to feed their expressed breast milk [2]. Other international agencies i.e., UNICEF, CDC, American Association of Pediatrics (AAP), Australian Breastfeeding Association and National bodies GOI, ICMR, NNF, IAP, FOSGI, IYCF Chapter of IAP recommended similar guidelines with respect to breastfeeding during the pandemic $[4-8,11,14,35]$. The UK Committee for UNICEF (UNICEF UK) Baby Friendly Initiative and Australian Breastfeeding Association have also updated their guidelines based on recent available evidence $[14,36]$.

Contributors: All authors were part of the IAP IYCF team that formulated the guidelines. $\mathrm{KB}, \mathrm{RMB}, \mathrm{ST}$, RS: conceived the guidelines, prepared the agenda, and executed administratively. KB and RMB: led the discussions and all the members actively participated. RMB, AB, AAK, HBM: reviewed the literature on national and international guidelines. $\mathrm{RMB}, \mathrm{KB}$ : wrote the first 
Table I Feeding Options for the Neonate Born to a Mother With Suspected/Confirmed COVID-19

\begin{tabular}{llll}
\hline Mother's clinical status & Clinical status of the baby & Rooming in & Feeding option \\
\hline $\begin{array}{l}\text { Mild COVID-19 } \\
\text { Asymptomatic or symptomatic }\end{array}$ & $\begin{array}{l}\text { Stable } \\
\text { Unstable }\end{array}$ & Yes & BF \\
Moderate COVID-19 & & NICU & EBM $>$ PDHM \\
Stable & Stable & Yes & BF \\
& Unstable & NICU & EBM $>$ PDHM \\
Unstable & Stable & Caretaker & EBM $>$ PDHM $>$ Wet nursing \\
& Unstable & NICU & EBM $>$ PDHM \\
Severe COVID-19a & & & \\
Unstable & Stable & Care taker & NICU \\
& Unstable & NICU & EBM $>$ PDHM $>$ Wet nursing \\
Death of the mother & Unstable & EBM (wet nurse's) $>$ PDHM
\end{tabular}

COVID-19: coronavirus disease 2019, BF: direct breastfeeding, EBM: expressed breast milk, PDHM: pasteurized donor human milk. The sign ' $>$ ' is used to show that the preceding option is the more preferred of the two.

draft. The first draft was peer reviewed by ST, RS, MP, UB, AB, JS, OK, DG, GVB, RK, PG: provided intellectual inputs and overall guidance at every step; PG, GVB, RK: provided the administrative support from the Indian Academy of Pediatrics (IAP) and coordinated between the team and executive board members of IAP. The final document was drafted and edited by $\mathrm{KB}$ and RMB. All authors approved the final recommendations of the guidelines.

Funding: None; Competing interest: None stated.

\section{REFERENCES}

1. WHO Coronavirus (COVID-19) Dashboard. Accessed June 8, 2021. Available from: https://covid19. who.int

2. WHO.2020. Breastfeeding advice during the COVID 19 outbreak. Accessed April 23, 2020. Available from: http:// www.emro.who.int/nutrition/nutrition-infocus/breastfeedingadvice-during-COVID-19-outbreak.html

3. UNICEF: Coronavirus Disease (COVID 19) information centre. Accessed June 8, 2021. Available from: https://www.unicef.org/ coronavirus/covid-19

4. Centres for Disease Control and Prevention, USA. Considerations for Inpatient Obstetric Healthcare Settings. Accessed May 20, 2020. Available from: https://www.cdc.gov/ coronavirus/2019cov/hcp/inpatient-obstetric-healthcareguidance.html

5. Ministry of Health and Family Welfare. Guidelines for Management of COVID 19. Accessed January 05, 2021. Available at https://www.mohfw.gov.in

6 ICMR-National Institute for Research in Reproductive Health 2020. Guidance for Management of Pregnant Women in COVID-19 Pan-demic. Accessed April 27, 2020. Available from:https://icmr.nic.in/sites/default/files/upload_documents/ Guidance_for_Management_of_Pregnant_Women_in_ COVID19 Pandemic 12042020.pdf

7. Chawla D, Kumar P, Mittal P, et al. Perinatal-neonatal management of COVID-19 infection, 2020. Indian Pediatr. 2020;57:536-48.

8. The Human Milk Bank Association of India, IAP-IYCF Chapter, PATH April 2020. Guidance for Use of Human Milk in the Context of COVID-19 in India. Accessed June 8, 2021. Available from: https://www.path.org/resources/ guidance-use-human-milk-india-context-covid-19

9. Update-on-COVID-19-and-Breastfeeding. Accessed June 8, 2021. Available from: https://www.bpni.org/covid-19-andbreastfeeding-resources/

10. Yeo KT , Oei J, De Luca D, et al. Review of guidelines and recommendations from 17 countries highlights the challenges that clinicians face caring for neonates born to mothers with COVID-19. Internat J Pediatr. 2020;109: 2192-207.

11. Wyckoff AS. AAP issues guidance on infants born to mothers with suspected or confirmed COVID19. Accessed June 62021. Available from: https://www.aappublications.org/news/2020/ 04/02/infant covid guidance040220

12. Sankar MJ, Neogi S, Sharma J, et al. State of newborn health in India. J Perinatol. 2016:36:S3-S8.

13. Perl SH, Uzan- Yulzari A, Klainer H , et al. SARS-CoV-2Specific antibodies in breast milk after COVID-19 vaccination of breast-feeding women. JAMA. 2021:325: 2013-14.

14. Unicef -Uk Baby -Friendly Initiative statement on infant feeding during Covid 19 outbreak. Accessed June 8 2021.Avaiable from: https://www.unicef.org.uk/baby friendly/ infant-feeding-during-the-covid-19

15. New Recommendations of NEGVAC accepted by Union Ministry of Health. Accessed May 21, 2021. Available from: https://pib.gov.in/PressReleasePage.aspx?PRID $=1719925$

16. Mustafa NM, Selim LA. Characterisation of COVID-19 pandemic in paediatric age group: A systematic review and metaanalysis. J Clin Virol. 2020;128:104395.

17. Vassilopoulou E, Feketea G, Koumbi L, et al. Breast-feeding and COVID-19: From nutrition to immunity. Front Immunol. 2021:12: 661806.

18. Vasques da Costa A, Purcell Goes C. Gama P. Breast-feeding importance and its therapeutic potential against SARS-CoV-2. Physiol Rep. 2021;9:e14744.

19. Lang J, Yang N, Deng J, et al. Inhibition of SARS pseudo virus cell entry by lactoferrin binding to heparan sulfate proteoglycans. PLoS One. 2011:6:e23710.

20. Rousseaux A, Brosseau C, Ie Gall S, et al. Human milk oligosaccharides: Their effects on the host and their potential as thera-peutic agents. Front Immunol. 2021:12: 680911.

21. Mitoulas LR, Schärer-Hernández NG, Liabat S. Breastfeeding, human milk and COVID-19-What does the evidence say? Front Pediatr. 2020;8:613339.

22. Pace RM, Williams JE, Jaarvinen KM, et al. COVID-19 and 
human milk: SARS-CoV-2, antibodies, and neutralizing capacity. MedRxiv [Preprint]. 2020:2020.09.16.20196071.

23. Fox A, Marino J, Amanat F, et al. Evidence of a significant secretory-IgA-dominant SARS-CoV-2 immune response in human milk following recovery from COVID-19. MedRxiv [Preprint]. 2020.

24. Lubbe W, Botha E, Niela-Vilen H, Reimers P. Breast-feeding during the COVID-19 pandemic - a literature review for clinical practice. Int Breastfeed J. 2020:15:82.

25. Rodrigues C, Baía I, Domingues R, Barros H. Pregnancy and breastfeeding during COVID-19 pandemic: A systematic review of published pregnancy cases. Front Publ Hlth. 2020;8:558144.

26. Hudak ML. Consequences of the SARS-CoV-2 pandemic in the perinatal period. Curr Op Pediatr. 2021:33:181-87.

27. Shlomai NO, Kasirer Y, Strauss T, et al. Neonatal SARS-CoV-2 infections in breastfeeding mothers. Pediatrics. 2021;147: e2020010918.

28. Bhatt H. Should COVID-19 mother breastfeed her newborn child? A literature review on the safety of breastfeeding for pregnant women with COVID-19. Curr Nutri Rep. 2021;10:7175.

29. Centeno-Tablante E, Medina-Rivera M, Finkelstein JL, et al. Transmission of SARS-CoV-2 through breast milk and breastfeeding: a living systematic review. Ann N Y Acad Sci. 2021;1484: $32-54$
30. Kumar J, Meena J, Yadav A, Kumar P. SARS-CoV-2 detection in human milk: a systematic review. J Matern Fetal Neonatal Med. 2021:1-8

31. Krogstad P, Contreras D, Ng H, et al. No evidence of infectious SARS-CoV-2 in human milk: analysis of a cohort of 110 lactating women. Medrxiv [Preprint]. 2021:2021.04.05.21254897

32. Chambers C, Krogstad P, Bertrand K, et al. Evaluation for SARS-CoV-2 in breast milk from 18 infected women. JAMA. 2020;324: 1347-48.

33. Kasi SG, Dhir SK, Shivananda S, et al. Breastfeeding and Coronavirus Disease 2019 (COVID-19) Vaccination: Position Statement of the Indian Academy of Pediatrics/Advisory Committee on Vaccination and Immunization Practices. Indian Pediatr. 2021;58:647-49.

34. Rollins N, Minckas N, Jehan F, et al. A public health approach for deciding policy on infant feeding and mother-infant contact in the context of COVID-19. Lancet Global Hlth. 2021;9:e55257.

35. Hull N, Kam RL, Gribble KD. Providing breastfeeding support during the COVID-19 pandemic: Concerns of mothers who contacted the Australian breastfeeding association. Breastfeeding Rev. 2020;28:25-35.

36. Australian breastfeeding association.COVID-19 vaccination and breastfeeding infographic final. Accessed June 8, 2021. Available from: https://www.breastfeeding.asn. au.bfinfo.covid-19

\section{NEWSIN BRIEF}

\section{Autism - The silent grabber of children}

Autism and Developmental Disabilities Monitoring (ADDM) Networkconducts active surveillance of ASD among children living in the areas under 11 ADDM sites across United States.In a recently published report, according to ADDM Network one in 44 children aged $\leq 8$ years were identified to have ASD, the incidence of which has increased markedly from 1 in 54 children over two years period. Other important findings - ASD was 4.2 times more prevalent among boys as compared to girls while $35 \%$ children were having an intelligence quotient (IQ) score $\leq 70$. Children having ASD with IQ score $\leq 70$ were diagnosed at an earlier age compared to those with IQ score $>70$.

Is this rise is a result of the early screening and referral or industrilization?As a primary care provider to the children / society we have to think about it. (Surveillance Summaries 03 December, 2021)

Rajesh Kumar Meena raj.mamc@gmail.com 